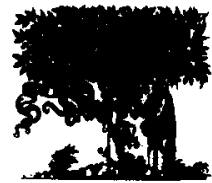

ELSEVIER

Signal Processing 46 (1995) 119-124

\title{
Fractional Fourier domains
}

\author{
Haldun M. Ozaktas*, Orhan Aytür \\ Department of Electrical Engineering. Bilkent University. TR-06533 Bilkent, Ankara. Turkey
}

Received 14 November 1994; revised 27 April 1995

\begin{abstract}
It is customary to define the time-frequency plane such that time and frequency are mutually orthogonal coordinates. Representations of a signal in these domains are related by the Fourier transform. We consider a continuum of "fractional" domains making arbitrary angles with the time and frequency domains. Representations in these domains are related by the fractional Fourier transform. We derive transformation, commutation, and uncertainty relations among coordinate multiplication, differentiation, translation, and phase shift operators between domains making arbitrary angles with each other. These results have a simple geometric interpretation in time-frequency space.
\end{abstract}

\section{Zusammenfassung}

Üblicherweise wird die Zeit-Frequenz-Ebene so definiert, daß Zeit und Frequenz orthogonale Koordinaten darstellen. Signaldarstellungen in diesen Bereichen ängen über die Fouriertransformation zusammen. Wir betrachten ein Kontinuum von "fraktionalen" Bereichen, die mit dem Zeitbereich und mit dem Frequenzbereich einen beliebigen Winkel einschließen. Signaldarstellungen in diesen Bereichen sind durch die "fraktionale Fouriertransformation" verknüpft. Wir zeigen Transformations-, Kommutations- und Unschärfebeziehungen von Koordinatenmultiplikations-, Differentiations-, Verschiebungs- und Phasenverschiebungsoperatoren zwischen Bereichen, die beliebige Winkel einschließen. Diese Ergebnisse erlauben eine einfache geometrische Interpretation im Zeit-Frequenz-Raum.

\section{Résumé}

Il est habituel de définir le plan temps-fréquence de telle sorte que le temps et la fréquence soient des coordonnées orthogonales. Les représentations d'un signal dans ces domaines sont reliées par la transformée de Fourier. Nous considérons un continuum de domaines "fractionnaires" faisant des angles arbitraires avec les domaines temporels et fréquentiels. Les représentations dans ces domaines sont reliées par la transformée de Fourier fractionnaire. Nous dérivons les rélations de transformation, de commutation et d'incertitude parmi les opérateurs de déplacement de phase, de translation, de différentiation, de multiplication de coordonnées entre des domaines faisant des angles arbitraires entre eux. Ces résultats ont une interprétation géométrique simple dans l'espace temps-fréquence.

Keywords: Fractional Fourier transforms; Time-frequency distributions; Wigner distribution

* Corresponding author. 
The purpose of this paper is to consolidate the concept of fractional Fourier domains suggested in a recent paper [16]. The present discussion will be framed in an operator formalism, whose power will be evident in deriving and representing a number of novel results.

Let $\psi$ denote a signal in the abstract, that is, without reference to any particular domain. This signal can be represented in the time domain by $\psi_{t}(t)$, or alternatively in the frequency domain by $\psi_{\mathrm{f}}(f)$, where $\psi_{\mathrm{f}}(f)$ is the Fourier transform of $\psi_{\mathrm{t}}(t)$. In this paper we discuss domains other than these two, which we call fractional Fourier domains. The representations of the signal in these domains are related to each other by the fractional Fourier transform.

The fractional Fourier transform $[1,9,14,15]$ has many applications in the solution of differential equations $[9,14]$, quantum mechanics $[23-25,27$, $28]$, diffraction theory and optical propagation, optical systems and signal processing $[2,3,6,12$, 18-22], swept-frequency filters [1], time-variant filtering and multiplexing $[5,13,16]$ neural networks [26] and study of time-frequency distributions [4]. It can be optically realized much like the usual Fourier transform, and has a fast digital algorithm $[5,15]$. We elaborate on these in the following paragraphs.

Linear differential equations with constant coefficients are used to represent linear time-invariant systems. These equations can be solved by taking their Fourier (or Laplace) transform. Differential equations representing time-variant systems, however, have nonconstant coefficients. At least certain classes of such equations can be solved by virtue of the additional degree of freedom afforded by the fractional Fourier transform $[9,14]$.

In quantum mechanics and optics, measurement of the phase of a signal is experimentally difficult. Knowledge of the Wigner distribution yields complete knowledge of the signal, but is difficult to measure directly. It is known that the amplitude squared fractional Fourier transform is the Radon transform of the Wigner distribution. This makes it possible to tomographically reconstruct the Wigner distribution by making a series of intensity measurements of the fractional transforms, if one knows how to obtain the fractional transforms of the signal [23-25]. This method should be generalizable to other problems where it is difficult to measure the phase of a signal, as is often the case with optical systems.

The fractional Fourier transform describes the propagation of optical wavefields through a rather general class of optical systems. The order of the fractional transform corresponds to the distance along the axis of propagation. The wavefields which are usually expressed in terms of complicated diffraction integrals can be more simply expressed in terms of the fractional Fourier transform [19-22]. This allows efficient computation of diffraction integrals and wavefields in optical systems. Also, it allows the properties of the wavefields to be deduced from the properties of the fractional Fourier transform. Furthermore, since the fractional Fourier transform can be implemented with the same amount of optical hardware as the ordinary Fourier transform $[2,3,6,10]$, the signal processing techniques discussed in the following paragraph can be implemented with optical systems.

The fractional Fourier transform can be calculated digitally in $\mathrm{O}(N \log N)$ time, just like the ordinary Fourier transform $[5,15]$. Ordinary Fourier domain filtering techniques are more suitable for time-invariant signals and systems. Filtering in fractional Fourier domains allows one to reduce the minimum-mean-square error in optimal filtering for the time-varying case $[5,16]$. In a similar spirit, multiplexing in fractional Fourier domains allows signals whose time-requency distribution is irregular to be packed more efficiently in a given channel [16].

In conclusion, the new perspective and analytical tools offered by the fractional Fourier transform and the concept of fractional Fourier domains in time-frequency space should prove fruitful for fundamental signal theory, and inspire many other applications and generalizations of existing methods wherever Fourier transforms are concerned. The purpose of this paper is to serve as a vehicle to this end.

Let $\mathscr{F}$ denote the Fourier transform operation so that $\psi_{1}(\cdot)=\mathscr{F}\left[\psi_{0}(\cdot)\right]$ is the Fourier transform of the function $\psi_{0}(\cdot)$. The ath order fractional Fourier 
transform operation is denoted as $\mathscr{F}^{a}$ so that $\psi_{a}(\cdot)=\mathscr{F}^{a}\left[\psi_{0}(\cdot)\right]$ is the ath-order fractional Fourier transform of the function $\psi_{0}(\cdot)$. $\mathscr{F}^{1}$ corresponds to the ordinary Fourier operation $\mathscr{F}$, and $\mathscr{F}^{0}$ is the identity operation. $\mathscr{F}^{2}$ corresponds to the coordinate reflection operation so that $\mathscr{F}^{4}$ is also equivalent to the identity operation. We also have $\mathscr{F}^{a_{1}} \mathscr{F}^{a_{2}}=\mathscr{F}^{a_{1}} \mid a_{2}$. The fractional Fourier transform can be defined for $0<|a|<2$ as [16]

$\psi_{a}(x)=\int_{-\infty}^{\infty} B_{a}\left(x, x^{\prime}\right) \psi_{0}\left(x^{\prime}\right) \mathrm{d} x^{\prime}$

$B_{a}\left(x, x^{\prime}\right)$

$$
=A_{\phi} \exp \left[\mathrm{i} \pi\left(x^{2} \cot \phi-2 x x^{\prime} \csc \phi+x^{\prime 2} \cot \phi\right)\right],
$$

$A_{\phi}=(|\sin \phi|)^{-1 / 2} \exp [\mathrm{i} \pi \operatorname{sgn}(\sin \phi) / 4-\mathrm{i} \phi / 2]$,

where $\phi=a \pi / 2$. The kernel $B_{a}\left(x, x^{\prime}\right)$ approaches $\delta\left(x-x^{\prime}\right)$ or $\delta\left(x+x^{\prime}\right)$ when $a$ approaches 0 or \pm 2 , respectively. The definition is easily extended outside the interval $[-2,2]$ by remembering that $\mathscr{F}^{4}$ is the identity operation [9].

The functions $\psi_{a}(\cdot)$ for different values of $a$ may be considered as different representations of the same signal $\psi$. In particular, $\psi_{0}\left(x_{0}\right)$ is the time domain representation of the signal, and $\psi_{1}\left(x_{1}\right)$ is the frequency domain representation $\left(\psi_{0}(\cdot)=\psi_{t}(\cdot)\right.$ and $\left.\psi_{1}(\cdot)=\psi_{\mathrm{f}}(\cdot)\right)$. We refer to the $x_{a}$ axis as the $a$ th fractional Fourier domain, and associate $x_{0}$ and $x_{1}$ with the conventional time and frequency axes $t$ and $f$, respectively.

There is nothing special about the $a=0$ representation, it merely corresponds to the choice of origin of the parameter $a$. We can transform from the $x_{a}$ representation to the $x_{a}$, representation by taking a fractional Fourier transform

$\psi_{a^{\prime}}\left(x_{a^{\prime}}\right)=\int B_{\left(a^{\prime}-a\right)}\left(x_{a^{\prime}}, x_{a}\right) \psi_{a}\left(x_{a}\right) \mathrm{d} x_{a}$.

When $a^{\prime}=a+1$, this is just an ordinary Fourier transformation.

We now introduce the family of coordinate-multiplication operators $\boldsymbol{X}_{a}$ parameterized by $a$. The operator $\boldsymbol{X}_{a}$ is defined to be such that, when it acts on the (abstract) signal $\psi$ from the left, its effect in the ath domain is

$\left\{\boldsymbol{X}_{a} \psi\right\}_{a}\left(x_{a}\right) \equiv x_{a} \psi_{a}\left(x_{a}\right)$.

Here $\left\{\boldsymbol{X}_{a} \psi\right\}_{a}(\cdot)$ denotes the representation of the signal $\boldsymbol{X}_{a} \psi$ in the ath domain. What is the effect of such an operator in another domain? For instance, what is the effect of the operator $\boldsymbol{X}_{a+1}$ in the ath domain? From the well known Fourier transform property stating that coordinate multiplication in the frequency domain corresponds to differentiation in the time domain, we obtain

$$
\left\{\boldsymbol{X}_{a+1} \psi\right\}_{a}\left(x_{a}\right)=\frac{1}{2 \pi \mathrm{i}} \frac{\mathrm{d}}{\mathrm{d} x_{a}} \psi_{a}\left(x_{a}\right) \equiv\left\{\boldsymbol{D}_{a} \psi\right\}_{a}\left(x_{a}\right),
$$

where the second equality defines the differentiation operator $\boldsymbol{D}_{a}$, which is seen to be equal to $\boldsymbol{X}_{a+1}$.

We now wish to express the operator $\boldsymbol{X}_{a}$ in terms of the operators $\boldsymbol{X}_{\boldsymbol{a}^{\prime}}$ and $\boldsymbol{X}_{\boldsymbol{a}+1}=\boldsymbol{D}_{\boldsymbol{a}^{\prime}}$ for any given value of $a^{\prime}$. The fractional Fourier transform property $[9,10]$

$$
\begin{aligned}
\mathscr{F}^{a^{\prime}-a} & {\left[x_{a} \psi_{a}\left(x_{a}\right)\right] } \\
= & x_{a^{\prime}}\left(\mathscr{F}^{a^{\prime}-a}\left[\psi_{a}\left(x_{a}\right)\right]\right) \cos \left(\phi^{\prime}-\phi\right) \\
& \quad-\quad \begin{array}{l}
\mathrm{d} \\
\left.\quad 2 \pi \mathrm{i} \frac{\mathrm{d} x_{a^{\prime}}}{\mathscr{F}^{a^{\prime}-a}}\left[\psi_{a}\left(x_{a}\right)\right]\right) \sin \left(\phi^{\prime}-\phi\right),
\end{array}
\end{aligned}
$$

can be derived directly from Eq. (2). Using this, we obtain

$X_{a}=X_{a^{\prime}} \cos \left(\phi^{\prime}-\phi\right)-X_{a^{\prime}+1} \sin \left(\phi^{\prime}-\phi\right)$.

Substituting $a \rightarrow a+1$ in Eq. (6) (or by using a property analogous to Eq. (5) for $\left.\mathscr{F}^{a^{\prime}-a}\left[\mathrm{~d} \psi_{a}\left(x_{a}\right) / \mathrm{d} x_{a}\right]\right)$ we obtain an equation for $X_{a+1}$, which when combined with Eq. (6) gives

$\left[\begin{array}{c}\boldsymbol{X}_{a} \\ \boldsymbol{X}_{a+1}\end{array}\right]=\left[\begin{array}{cr}\cos \left(\phi^{\prime}-\phi\right) & -\sin \left(\phi^{\prime}-\phi\right) \\ \sin \left(\phi^{\prime}-\phi\right) & \cos \left(\phi^{\prime}-\phi\right)\end{array}\right]\left[\begin{array}{c}\boldsymbol{X}_{a^{\prime}} \\ \boldsymbol{X}_{a^{\prime}+1}\end{array}\right]$.

(We can replace $\boldsymbol{X}_{a+1}=\boldsymbol{D}_{a}$ and $\boldsymbol{X}_{a^{\prime}+1}=\boldsymbol{D}_{a^{\prime}}$ if we wish). This equation suggests an analogy with basis vectors in $\boldsymbol{R}^{2}$. Referring to Fig. 1, we see that the angle $\phi=a \pi / 2$ may be interpreted as the angle the ath domain makes with the 0th (position or space) domain and $\left(\phi^{\prime}-\phi\right)=\left(a^{\prime}-a\right) \pi / 2$ may be interpreted as the angle between the $a$ th and $a^{\prime}$ th domains. In particular, domains whose indices 


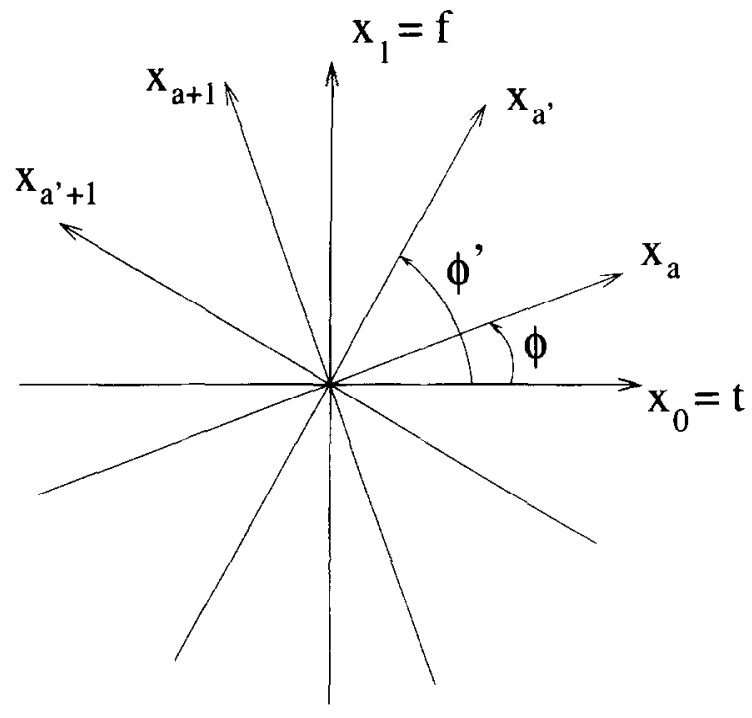

Fig. 1.

differ by unity are orthogonal. (This is consistent with the customary definition of phase space such that $t$ and $f$ are mutually orthogonal coordinates.)

The $\boldsymbol{X}_{a}$ operator, which simply multiplies $\psi_{a}\left(x_{a}\right)$ by $x_{a}$ in the $a$ th domain, results in a linear combination of multiplication by $x_{a^{\prime}}$ and differentation with respect to $x_{a^{\prime}}$ in the $a^{\prime}$ th domain. The $X_{a+1}$ operator, which simply multiplies by $x_{a+1}$ in the $(a+1)$ th domain, and which differentiates with respect to $x_{a}$ in the ath domain, results in a similar linear combination. The coefficients of these linear combinations are cosines and sines corresponding to the 'projection' of these operators on the $a^{\prime}$ th domain.

It is also possible to express $\boldsymbol{X}_{\boldsymbol{a}}$ in terms of any two operators $\boldsymbol{X}_{a^{\prime}}$ and $\boldsymbol{X}_{a^{\prime \prime}}$, provided the latter two are not collinear, and express any function of an arbitrary number of operators $F\left(X_{b^{\prime}}, X_{b^{\prime \prime}}, \ldots\right)$ in the form $F^{\prime}\left(\boldsymbol{X}_{a^{\prime}}, \boldsymbol{X}_{a^{\prime \prime}}\right)$. Furthermore, it is possible to define the space spanned by any two noncollinear operators $\boldsymbol{X}_{a}$ and $\boldsymbol{X}_{a^{\prime}}$, and operations such as inner products and norms. Although we do not present these extensions here, we will further discuss the above geometrical interpretation at the end of this paper in conjunction with Wigner distributions.
The commutator between two arbitrary operators $\boldsymbol{X}_{a}$ and $\boldsymbol{X}_{a^{\prime}}$, denoted by square brackets, is

$$
\left[\boldsymbol{X}_{a}, \boldsymbol{X}_{a^{\prime}}\right] \equiv \boldsymbol{X}_{a} \boldsymbol{X}_{a^{\prime}}-\boldsymbol{X}_{a} \cdot \boldsymbol{X}_{a}=\frac{\mathrm{i}}{2 \pi} \sin \left(\phi^{\prime}-\phi\right) .
$$

The first equality is the definition of the commutator, and the second is derived using Eqs. (4) and (7) and the well known commutator $\left[\boldsymbol{X}_{0}, \boldsymbol{X}_{1}\right]=\left[\boldsymbol{X}_{\mathrm{t}}, \boldsymbol{X}_{\mathrm{f}}\right]=\mathrm{i} / 2 \pi$. Now, using a standard result which applies when the commutator of two operators is a scalar quantity [8], this commutation relation between two nonorthogonal domains implies the uncertainty relationship

$$
\operatorname{var}\left[\psi_{a}\left(x_{a}\right)\right] \times \operatorname{var}\left[\psi_{a^{\prime}}\left(x_{a^{\prime}}\right)\right] \geqslant \frac{1}{16 \pi^{2}} \sin ^{2}\left(\phi^{\prime}-\phi\right)
$$

between representations in these two domains. Here var denotes the variance of the functions. The existence of such an uncertainty relationship was previously conjectured in [16]. Of course, the above simplifies to the well-known relationship between a function and its Fourier transform when $\phi^{\prime}-\phi$ is an odd multiple of $\pi / 2$.

We now define the linear phase operator $\boldsymbol{P}_{a}(\xi) \equiv \exp \left(\mathrm{i} \xi \boldsymbol{X}_{a}\right)$. Its effect in the ath domain is given simply by $\left\{\exp \left(\mathrm{i} \xi \boldsymbol{X}_{a}\right) \psi\right\}_{a}\left(x_{a}\right)=\exp \left(\mathrm{i} \xi x_{a}\right) \times$ $\psi_{a}\left(x_{a}\right)$, as can be verified by series expansion of the exponential. On the other hand, the effect of the $\exp \left(i \xi X_{a+1}\right)$ operator in the same domain is given by

$$
\left\{\mathrm{e}^{\mathrm{i} \xi \boldsymbol{X}_{a+1}} \psi\right\}_{a}\left(x_{a}\right)=\psi_{a}\left(x_{a}+\xi\right) \equiv\left\{\boldsymbol{T}_{a}(\xi) \psi\right\}_{a}\left(x_{a}\right) .
$$

This is just the Fourier transform property stating that multiplication by a phase factor in one domain corresponds to translation in the orthogonal domain. The second equality defines the translation operator $\boldsymbol{T}_{a}(\xi)$, which is seen to be equal to $\exp \left(\mathrm{i} \xi \boldsymbol{X}_{u+1}\right)=\exp \left(\mathrm{i} \xi \boldsymbol{D}_{a}\right)$. Using Eq. (7), the operator $\exp \left(\mathrm{i} \xi \boldsymbol{X}_{a}\right)$ can be expressed in the $a^{\prime}$ th domain as

$$
\begin{aligned}
\mathrm{e}^{\mathrm{i} \xi X_{a}}= & \mathrm{e}^{-\mathrm{i} \xi^{2} \sin \left(\phi^{\prime}-\phi\right) \cos \left(\phi^{\prime}-\phi\right) / 2} \\
& \times \mathrm{e}^{\mathrm{i} \xi X_{a^{\prime}} \cos \left(\phi^{\prime}-\phi\right)} \mathrm{e}^{-\mathrm{i} \xi \boldsymbol{X}_{a^{\prime}+1} \sin \left(\phi^{\prime}-\phi\right)} \\
= & \mathrm{e}^{\mathrm{i} \xi^{2} \sin \left(\phi^{\prime}-\phi\right) \cos \left(\phi^{\prime}-\phi\right) / 2} \\
& \times \mathrm{e}^{-\mathrm{i} \xi X_{a^{\prime}+1} \sin \left(\phi^{\prime}-\phi\right)} \mathrm{e}^{\mathrm{i} \xi X_{a^{\prime}} \cos \left(\phi^{\prime}-\phi\right)},
\end{aligned}
$$


where Glauber's formula [8] $\exp (\boldsymbol{A}+\boldsymbol{B})=\exp (\boldsymbol{A}) \times$ $\exp (\boldsymbol{B}) \exp (-[\boldsymbol{A}, \boldsymbol{B}] / 2)$ for any two operators $\boldsymbol{A}$ and $\boldsymbol{B}$ that commute with their commutator has been used. Rewriting the same equation in terms of the phase and translation operators we obtain

$$
\begin{aligned}
\boldsymbol{P}_{a}(\xi)= & \mathrm{e}^{-\mathrm{i} \xi^{2} \sin \left(\phi^{\prime}-\phi\right) \cos \left(\phi^{\prime}-\phi\right) / 2} \\
& \times \boldsymbol{P}_{a^{\prime}}\left(\xi \cos \left(\phi^{\prime}-\phi\right)\right) \boldsymbol{T}_{a^{\prime}}\left(-\xi \sin \left(\phi^{\prime}-\phi\right)\right) \\
= & \mathrm{e}^{\mathrm{i} \xi^{2} \sin \left(\phi^{\prime}-\phi\right) \cos \left(\phi^{\prime}-\phi\right) / 2} \\
& \times \boldsymbol{T}_{a^{\prime}}\left(-\xi \sin \left(\phi^{\prime}-\phi\right)\right) \boldsymbol{P}_{a^{\prime}}\left(\xi \cos \left(\phi^{\prime}-\phi\right)\right) .
\end{aligned}
$$

We see that the $\boldsymbol{P}_{a}(\xi)=\exp \left(\mathrm{i} \xi \boldsymbol{X}_{a}\right)$ operator, which simply results in a phase shift in the ath domain, results in a translation followed by a phase shift (or a phase shift followed by a translation) in the $a^{\prime}$ th domain. (The fact that translation and phase shifting do not commute accounts for the additional phase factor coming from Glauber's formula.) By employing the substitution $a \rightarrow a+1$, we can obtain a second pair of equations similar to Eq. (11):

$$
\begin{aligned}
\mathrm{e}^{\mathrm{i} \xi X_{a^{+1}}=} & \mathrm{e}^{\mathrm{i} \xi^{2} \sin \left(\phi^{\prime}-\phi\right) \cos \left(\phi^{\prime}-\phi\right) / 2} \\
& \times \mathrm{e}^{\mathrm{i} \xi \boldsymbol{X}_{a^{\prime}} \sin \left(\phi^{\prime}-\phi\right)} \mathrm{e}^{\mathrm{i} \xi \boldsymbol{X}_{a^{\prime}+1} \cos \left(\phi^{\prime}-\phi\right)} \\
= & \mathrm{e}^{-\mathrm{i} \xi_{\xi^{2}} \sin \left(\phi^{\prime}-\phi\right) \cos \left(\phi^{\prime}-\phi\right) / 2} \\
& \times \mathrm{e}^{\mathrm{i} \xi \boldsymbol{X}_{a^{\prime}+1} \cos \left(\phi^{\prime}-\phi\right)} \mathrm{e}^{\mathrm{i} \xi X_{a^{\prime}} \sin \left(\phi^{\prime}-\phi\right)}
\end{aligned}
$$

or

$$
\begin{aligned}
\boldsymbol{T}_{a}(\xi)= & \mathrm{e}^{\mathrm{i} \xi^{2} \sin \left(\phi^{\prime}-\phi\right) \cos \left(\phi^{\prime}-\phi\right) / 2} \\
& \times \boldsymbol{P}_{a^{\prime}}\left(\xi \sin \left(\phi^{\prime}-\phi\right)\right) \boldsymbol{T}_{a^{\prime}}\left(\xi \cos \left(\phi^{\prime}-\phi\right)\right) \\
= & \mathrm{e}^{-\mathrm{i} \xi^{2} \sin \left(\phi^{\prime}-\phi\right) \cos \left(\phi^{\prime}-\phi\right) / 2} \\
& \times \boldsymbol{T}_{a} \cdot\left(\xi \cos \left(\phi^{\prime}-\phi\right)\right) \boldsymbol{P}_{a} \cdot\left(\xi \sin \left(\phi^{\prime}-\phi\right)\right) .
\end{aligned}
$$

We see that $\boldsymbol{T}_{a}(\xi)=\exp \left(\mathbf{i} \xi \boldsymbol{X}_{a+1}\right)$ operator, which simply results in a translation in the ath domain, results in a translation followed by a phase shift (or a phase shift followed by a translation) in the $a^{\prime}$ th domain. As with Eq. (11), here also the amount of translation and phase shift is given by cosine and sine multipliers corresponding to the projection of the translation or phase shift on the new set of axes. It is also worth noting that starting from Eqs. (11) and (13) we can obtain formulas similar to Eq. (5) for $\mathscr{F}^{a^{\prime}-a}\left[\exp \left(\mathrm{i} \xi x_{a}\right) \psi_{a}\left(x_{a}\right)\right]$ and
$\mathscr{F}^{a^{-}-a}\left[\psi_{a}\left(x_{a}-\xi\right)\right]$. (These formulas can also be derived directly from Eq. (1) $[1,9,10]$.)

In passing we underline that all of the four different operators $\boldsymbol{X}_{a}, \boldsymbol{D}_{a}, \boldsymbol{P}_{a}(\xi)$ and $\boldsymbol{T}_{a}(\xi)$ are expressible in terms of the basic operator $\boldsymbol{X}_{\boldsymbol{a}}$.

Everything derived until now strongly supports the analogy depicted in Fig. 1. Finally, we discuss how this is directly related to an important property of fractional Fourier transforms. The Wigner distribution $W_{\psi}(t, f)$ of $\psi$ is given by [16]

$$
\begin{gathered}
W_{\psi}(t, f)=\int_{-\infty}^{\infty} \psi_{t}\left(t+t^{\prime} / 2\right) \psi_{t}^{*}\left(t-t^{\prime} / 2\right) \\
\times \exp \left(-2 \pi \mathrm{i} f t^{\prime}\right) \mathrm{d} t^{\prime}
\end{gathered}
$$

The Wigner distribution can be equally well defined in terms of the representation $\psi_{a}\left(x_{a}\right)$ of the signal $\psi$ in any domain $a$.

It is possible to relate $\psi_{a}\left(x_{a}\right)$ to the Wigner distribution by $[7,12,16]$

$\mathscr{R}_{\phi}\left[W_{\psi}(t, f)\right]=\left|\psi_{a}\left(x_{a}\right)\right|^{2}$,

where the Radon transform operation $\mathscr{R}_{\phi}$ takes the integral projection of the Wigner distribution on an axis making angle $\phi$ with the $x_{0}=t$ axis. Two widely known special cases are

$$
\begin{aligned}
& \int W(t, f) \mathrm{d} f=\left|\psi_{t}(t)\right|^{2}, \\
& \int W(t, f) \mathrm{d} t=\left|\psi_{f}(f)\right|^{2} .
\end{aligned}
$$

Eq. (16) means that the projection of the Wigner distribution of $\psi$ on an axis making angle $\phi=a \pi / 2$ with the $x_{0}=t$ axis gives the absolute square of the representation of $\psi$ in the ath domain. This supports the idea of referring to the axis making an angle $\phi$ with the $x_{0}$ axis as the $x_{a}$ axis (or the $a$ th domain), as depicted in Fig. 1 [16].

We now summarize. We speak of two representations which are related through a Fourier transform as being orthogonal to each other. The operator $\boldsymbol{X}_{a}$ is orthogonal to the operator $\boldsymbol{X}_{a+1}$, or equivalently, the operation of multiplying by $x_{a}$ is orthogonal to the operation $\mathrm{d} / \mathrm{d} x_{a}$. Likewise, multiplication by a phase factor is orthogonal to a corresponding translation, and so forth. In general, two representations that are related through a 
fractional Fourier transform of order $a$ make an angle $\phi=a \pi / 2$ with each other. Coordinate multiplication or differentiation in one of these domains results in a combination of these two operations in the other domain, as given by Eq. (7). Likewise, multiplication by a phase factor or a translation in one of these domains results in a combination of these two operations in the other domain, as given by Eqs. (11) and (13). The weighting factors appearing in these equations are cosines and sines with a direct interpretation as projections. The commutator and uncertainty relation between nonorthogonal domains are also interpreted in terms of this geometric picture.

\section{References}

[1] L.B. Almeida, "The fractional Fourier transform and timefrequency representations", IEEE Trans. Signal Process., Vol. 42, 1994, pp. 3084-3091.

[2] L.M. Bernardo and O.D.D. Soares, "Fractional Fourier transforms and optical systems", Opt. Commun., Vol. 110, 1994, pp. 517-522.

[3] L.M. Bernardo and O.D.D. Soares, "Fractional Fourier transforms and imaging", J. Opt. Soc. Amer. A, Vol. 11, 1994, pp. 2622-2626.

[4] J.R. Fonollosa and C.L. Nikias, "A new positive timefrequency distribution", Proc. IEEE Internat. Conf. Acoustics Speech Signal Process., 1994, pp. IV-301IV-304.

[5] M.A. Kutay, H.M. Ozaktas, L. Onural and O. Arikan, "Optimal filtering in fractional Fourier domains", Proc. IEEE Internat. Conf. Acoustics Speech Signal Process., 1995. IEEE Trans. Signal Process., Submitted.

[6] A.W. Lohmann, "Image rotation, Wigner rotation and the fractional Fourier transform", J. Opt. Soc. Amer. A, Vol. 10, 1993, pp. $2181-2186$.

[7] A.W. Lohmann and B.H Soffer, "Relationship between the Radon-Wigner and fractional Fourier transforms", J. Opt. Soc. Amer. A, Vol. 11, 1994, pp. 1798-1801.

[8] W.H. Louisell, Quantum Statistical Properties of Radiation, Wiley, New York, 1973.

[9] A.C. McBride and F.H. Kerr, "On Namias's fractional Fourier transform" IMA J. Appl. Math., Vol. 39, 1987, 159-175.

[10] D. Mendlovic and II.M Ozaktas, "Fractional Fourier transformations and their optical implementation: Part I", J. Opt. Soc. Amer. A, Vol. 10, 1993, pp. 1875-1881.

[11] D. Mendlovic, H.M Ozaktas and A.W. Lohmann, "Fourier transforms of fractional order and their optical interpretation", in: Topical Meeting on Optical Computing, OSA Technical Digest Series, Optical Society of America, Washington, DC, 1993, pp. 127-130.
[12] D. Mendlovic, H.M. Ozaktas and A.W. Lohmann, "Graded-index fibers, Wigner-distribution functions, and the fractional Fourier transform", Appl. Opt., Vol. 33, 1994, pp. 6188-6193.

[13] D. Mendlovic, H.M. Ozaktas and A.W. Lohmann, "Fractional correlation", Appl. Opt., Vol. 34, 1995, pp. 303-309.

[14] V. Namias, "The fractional Fourier transform and its application in quantum mechanics", J. Inst. Math. Appl., Vol. 25, 1980, pp. 241-265.

[15] H.M. Ozaktas, O. Arikan, M.A. Kutay and G. Bozdaği, "Digital computation of the fractional Fourier transform", IEEE Trans. Signal Process., submitted.

[16] H.M. Ozaktas, B. Barshan, D. Mendlovic and L. Onural, "Convolution, filtering, and multiplexing in fractional Fourier domains and their relation to chirp and wavelet transforms", J. Opt. Soc. Amer. A, Vol. 11, 1994, pp. 547-559.

[17] H.M. Ozaktas and D. Mendlovic, "Fourier transforms of fractional order and their optical interpretation", Opt. Commun., Vol. 101, 1993, pp. 163-169.

[18] H.M. Ozaktas and D. Mendlovic, "Fractional Fourier transformations and their optical implementation: Part II", J. Opt. Soc. Amer. A, Vol. 10, 1993, pp. 2522-2531.

[19] H.M. Ozaktas and D. Mendlovic, "The fractional Fourier transform as a tool for analyzing beam propagation and spherical mirror resonators", Opt. Lett., Vol. 19, 1994, pp. $1678-1680$.

[20] H.M. Ozaktas and D. Mendlovic, "Fractional Fourier optics", J. Opt. Soc. Amer. A., Vol. 12, 1995, pp. 743-751.

[21] P. Pellat-Finet, "Fresnel diffraction and the fractionalorder Fourier transform", Opt. Lett., Vol. 19, 1994, pp. 1388-1390.

[22] P. Pellat-Finet and G. Bonnet, "Fractional-order Fourier transform and Fourier optics", Opt. Commun., Vol. 111 1994, pp. 141-154.

[23] M.G. Raymer, M. Beck and D.F. McAlister, "Complex wave-field reconstruction using phase-space tomography", Phys. Rev. Lett., Vol. 72, 1994, pp. 1137-1140.

[24] M.G. Raymer, M. Beck and D.F. McAlister, "Spatial and temporal optical field reconstruction using phase-space tomography", J.D. Harvey and D.F. Wall, eds, Quantum Optics VI, Springer, Berlin, 1994.

[25] D.T. Smithey, M. Beck and M.G. Raymer, "Measurement of the Wigner distribution and the density matrix of a light mode using optical homodyne tomography: Application to squeezed states and the vacuum", Phys. Rev. Lett., Vol. 70, 1993, pp. 1244-1247.

[26] Soo-Young Lee and H.H. Szu, "Fractional Fourier transforms, wavelet transforms, and adaptive neural networks", Opt. Engrg., Vol. 33, 1994, pp. 2326-2330.

[27] K. Vogel and H. Risken, "Determination of quasiprobability distributions in terms of probability distributions for the rotated quadrature phase", Phys. Rev. A, Vol. 40, 1989, pp. 2847-2849.

[28] B. Yurke, W. Schleich and D.F. Walls, "Quantum superposition generated by quantum nondemolition measurements", Phys. Rev. A, Vol. 42, 1990, pp. 1703-1711. 